\title{
Le Dit d'un griot natif du Québec, fou d'Afrique ${ }^{1}$
}

\author{
Robert Viau \\ Université du Nouveau-Brunswick
}

Au fond des êtres je m'abrite

Dans leur sommeil je cherche repos

Dans leur regard chaude lumière

Dans leurs mains le vrai hameau. ${ }^{2}$

$S$ uccessivement professeur d'histoire et directeur du Centre d'études canadiennesdu Québec à Paris, secrétaire général de l'Agence de coopération culturelle et technique (devenue l'Agence de la Francophonie) pendant deux mandats successifs, Jean-Louis Roy a effectué un parcours qui l'a amené à s'éloigner du Québec et à écrire au sujet d'autres continents, d'autres civilisations. De Maîtres chez nous (1968) à Lumières d'Afrique (1997), de La Beauceronne, Marie à Georges à Joseph (1977) à La Francophonie: l'émergence d'une alliance (1989), de Programmes électoraux du Québec (1970) à La Mondialisation: développement et culture (1995), Jean-Louis Roy, sans renier ses origines, a ouvert le dialogue entre le Québec et la Francophonie et a enrichi la littérature québécoise de thèmes nouveaux qui sortent du cadre laurentien. Diplomate toujours avide de connaître, il a été amené de par ses fonctions à se rendre en des contrées éloignées, aux beaux noms exotiques qui n'appartiennent pas à nos civilisations de l'Occident. Il a recueilli attentivement la parole et les récits de l'Autre, qu'il soit président de république ou fellah. Ces visites et ces rencontres lui ont servi, comme à tout romancier, à la composition de son oeuvre, mais celle-ci se démarque par un réel respect des coutumes et des moeurs des sociétés qu'il a fréquentées. Il ne s'agit aucunement dans ses textes d'imposer la vision d'un «touriste québécois» ou celle d'un Blanc en tournée dans le TiersMonde, mais d'aller vers l'Autre afin de le connaître dans toute sa différence.

Dans Le Pèlerin noir $(1997)^{3}$, Jean-Louis Roy met à contribution sa formation d'historien, son expérience de diplomate et son talent de poète et de romancier ${ }^{4}$ afin de nous 
faire part de son attachement pour l'Afrique. Il nous rappelle que tandis qu'au début du quatorzième siècle, l'Europe s'engouffrait dans une période de crise générale, était mise à feu et à sang par la guerre de Cent Ans et autres conflits «locaux», dévastée par la peste, affaiblie par les famines et la pauvreté, l'Afrique et ce que nous nommons le Proche-Orient (ce qui est une désignation contestée, car proche de quoi?) connaissaient un âge d'or. Le livre de JeanLouis Roy reconstitue une histoire singulière, celle du pèlerinage à La Mecque, en 1323-1324, d'un jeune aristocrate africain, le Kankan Moussa, roi de Niani, avec une suite de plus de huit mille hommes, «douze mille selon certains» (9). Ce pèlerinage permet au souverain de l'Empire du Mali de rencontrer et de discuter avec les gouverneurs des États qu'il traverse et avec les hommes de science et les hommes de foi les plus illustres du temps. En cours de route, il rencontre des pèlerins venus de Marrakech, de Tunis, du Caire, de Jérusalem, de Bagdad, d'Ispahan et de l'Inde lointaine; il explore l'état du monde, se fait expliquer les technologies nouvelles et interroge les systèmes politiques. Le pèlerinage du roi de Niani nous fait découvrir ce que nous ne connaissons pas ou feignons de ne pas connaître, car allant à l'encontre des idées reçues et de notre ethnocentrisme, que le centre de la civilisation au quatorzième siècle était le monde musulman.

Quelques éléments de ce pèlerinage «sont connus avec certitude» (9) nous annonce l'auteur, mais la majorité des événements décrits ont été reconstruits avec patience et beaucoup de soin, la longue liste bibliographique, qui «n'est pas exhaustive» (175), en faisant foi. Mais comment rendre compte de la vie politique et de la cour du roi à cette époque? Pour décrire les relations entre les États, les négociations et les échanges entre les puissances, le romancier tire avantage non seulement de ses connaissances livresques, mais encore de son expérience du monde et des hommes. Jean-Louis Roy met à profit les années passées dans le service diplomatique. Ainsi, dans son récit, il décrit en détail comment les chefferies des peuples formées en délégations sont reçues au Palais selon «un protocole ancien, simple et majestueux» (12). Tout est réglementé, les cérémonies, l'ordre de préséance, les discours, jusqu'aux présents offerts au roi. De même, si le voyage du Kankan Moussa est avant tout un pèlerinage religieux, c'est aussi une série de «sommets» politiques qui permettent au souverain de «conforter ou établir des liens et des accords féconds» $(25-26)$ avec de puissants royaumes. Le chef du clan des Condé reçoit du Roi le mandat de planifier le voyage du souverain; il agit en quelque sorte à titre de ce que nous nommons de nos jours un «sherpa»: «arrêter l'agenda des rencontres, fixer les résidences royales et les lieux de séjour de son abondante suite, et régler les questions de sécuritề» (27). Le tout relève d'une connaissance profonde du cérémonial diplomatique.

Au niveau de la langue, l'auteur réussit à dépayser le lecteur par un dosage savant de mots et d'expressions étrangers, mais courants en Afrique: acheb, balafon, bâtas, bembé, cauri, cora, griot, hammam, igname, khamsa, takchif, thailécam, etc. On nous rappelle constamment que ce roi règne sur un pays désertique, où même les vents sont différents des nôtres: «Ces grandes

\section{LittéRéalité 80}


couvertures doivent protéger les voyageurs de l'harmattan, ce vent d'Est, sec et violent, ou de l'alizé, ce vent froid venu du Nord, des vents de sable aussi, cet opaque rideau de poussière chaude» (37). Des faits inexplicables, qui relèvent de l'imaginaire des peuples du désert, sont racontés: «ces forêts sacrées se déplaçant pour suivre leurs peuples; ces rivières s'enroulant comme une peau de serpent pour former sources et points d'eau; ces pêches miraculeuses faites dans des étangs grands comme l'ombre d'une cora; ces animaux venus combattre l'ennemi» (14). Les griots, ces bibliothèques humaines, retracent l'origine des peuples, les ancêtres des rois, les alliances stratégiques, les guerres gagnées ou perdues, tous les événements marquants, «fixé[s] à jamais dans la mémoire, ce récipient de tous les pardons et de toutes les ripostes» (15). Des vers du Coran, d'Abi Ibn Sayd, d'Antara ou Ibn Shaddâd ibn Qurâd Al-'Absî, d'Oumayya Ibn Abi'l-Salt, d'Al Farazdaq, d'Ibn Al-Roumi ou d'Ali Al-Baghdadi sont cités.

\author{
La terre, il l'a étendue \\ En surface plane \\ Et de lumière il l'a ornée \\ Soleil qui éclaire \\ Et croissant de lune \\ Et brassées d'étoiles \\ Qui, dans sa nuit sombre \\ Étincellent en perles \\ Et lancent des flèches \\ De feu (66)
}

À mesure que le Kankan Moussa et sa suite s'enfoncent dans le désert «avec ses couleurs particulières, ses longues plaines et ses bas plateaux, ses sols sableux et ses surfaces limoneuses» (74), le lecteur pénètre dans une culture dont il ne soupçonnait ni la richesse ni la diversité. Que ce soit par la description des contrées traversées ou par celle que font des voyageurs de Fès ou de Kairouan, le lecteur découvre dans toute sa beauté et son ampleur le monde islamique et africain.

Le Kankan Moussa a quitté son pays pour apprendre en voyant, mais aussi pour «revoir» ce qu'il a lu dans ce Coran avec lequel il a une incroyable familiarité. S'il traverse les déserts, c'est pour accueillir le privilège de «l'intime amitié de Dieu» (Coran, IV, 25). Il sait les risques de cette quête d'absolu, mais il l'entreprend pour ne pas perdre l'essentiel. Il opte pour la dépossession et pour cette expérience de mort au désert, afin de lutter contre la perte de spiritualité qui minait sournoisement sa vie quotidienne. Après avoir traversé le désert du Sahara et le désert d'Arabie, un lieu ardu et plein de risques, il contemple le lieu saint où repose Mahomet, la mosquée Haram et la Ka'bah, et dira après son retour à Niani que «la victoire de 
la vie sur la mort est une évidence, la primauté de l'accomplissement sur l'anéantissement, une même évidence» (145). Il revient du saint pèlerinage, le «Hajj», l'un des cinq préceptes fondamentaux du Prophète, plus aguerri, plus sage. Le pèlerin noir a surtout l'impression d'avoir entrevu une autre qualité d'existence, d'avoir progressé sur la voie de l'esprit, car «le pèlerinage purifie les coeurs et les esprits. Il constitue l'initiation la plus haute, celle qui conduit à la compréhension de la volonté supérieure, le lien vital entre notre regard et la lumière intérieure» (21). Le pèlerin a accédé à un mode d'être se rapprochant d'une conformité à la volonté créatrice.

La difficulté de faire revivre une telle époque et une telle civilisation est surmontée par un savant mélange de vérités historiques et d'inventions romanesques, de descriptions et de narrations. À mesure que progresse le pèlerinage du roi, l'auteur nous fait découvrir la situation politique du royaume de Niani, le rituel de chaque journée de marche dans le désert, il nous explique l'importance des oasis et des palmiers dans les ergs et les hamadas. Le tout prend une coloration pédagogique et nous croyons entendre un professeur d'histoire reconverti en romancier. Mais tel est le but de l'auteur, car joignant l'utile à l'agréable, il force le lecteur à revoir ses jugements face à ces pays du Sahel. L'auteur de ce récit singulier est effectivement un homme du monde. Cela se voit dans l'intérêt qu'il porte aux systèmes politiques, aux fastes des différentes cours, etc. Mais il est surtout un homme ouvert sur le monde. Porté vers l'Autre et l'Ailleurs, il décrit avec une curiosité bienveillante tout ce que le Kankan Moussa a pu arriver à connaître. Il rapporte le mode de vie des autres, le cadre de vie ailleurs. Les civilisations étrangères ne le laisse pas indifférent. Son récit a de passionnant le fait qu'on y trouve une profusion de détails historiques et géographiques, et le fait que l'auteur traite ses personnages de races et de croyances différentes de la même manière, avec la même tolérance et une compréhension rare. On comprend dès lors que le récit du pèlerinage soit beaucoup plus qu'un journal de voyage. C'est un itinéraire allégorique, un désir de connaissance de «ces hommes noirs venus d'un autre monde» (38). Ce faisant, Jean-Louis Roy fait sien ce proverbe peul placé en tête de son ouvrage: «Puisse ton action avoir un effet comparable à celui de la graine de baobab» (7). Le long voyage que décrit Jean-Louis Roy fait découvrir aux lecteurs de nouveaux pays et élargit leur horizon en les confrontant à d'autres paysages, d'autres cultures, d'autres hommes.

Dans un deuxième récit, Lumières d'Afrique ${ }^{5}$, Jean-Louis Roy prend la parole à son compte, sans l'intermédiaire d'un personnage fictif, et raconte des expériences et des découvertes personnelles. À titre de secrétaire général de l'Agence de coopération culturelle et technique, il a visité régulièrement les pays africains du nord au sud, de l'est à l'ouest. Dans son livre, il accomplit en quinze chapitres un périple qui va de l'Afrique aux Afriques, «en passant par des lieux du littoral et de l'intérieur, par des villes et des déserts, à travers des foules palabreuses et des solitudes on croirait du premier matin des éléments, des êtres et des choses» 
(7). Cependant, il ne s'agit pas de rédiger un récit de voyage pour les bibliothèques de gare ou pour l'agence Cook. L'auteur récuse une certaine variété d'exotisme, fondée sur les différences superficielles, celles liées au climat, au paysage, à la nature, au profit de l'étude de l'homme, des sociétés et des cultures.

Cet écrivain «fou d'Afrique» (7), comme on dirait fou d'amour pour reprendre le mot $\mathrm{d}$ 'André Breton, va de Dakar où il interroge le poète Amadou Lamine Sall qui «circule dans le sens et dans le langage avec la ferveur du vigneron dans ses chais») (22-23), à Tunis où le cinéaste Nacer Khmir «inventorie les soixante définitions de l'amour» (34). Il évoque l'ancien et le moderne: Ouidah, au Bénin, tout en mouvement vaudou, où des descendants d'esclaves venus des États-Unis, des Antilles, d'Haïti et du Brésil satisfont «leur besoin incoercible de communion [et] cette alliance de toute chose: dieux, morts, nature, comme un tout intangible et absolu» (39 et 41), puis Abidjan et la première édition du Marché des Arts du Spectacle Africain (MASA), où Wèrè Wèrè Liking invente un nouveau théâtre africain avec «les corps [...] enveloppés dans les matériaux de l'âme» (48). Du musée national de Nouakchott, la maison des nomades et de la mémoire du Sahara, il se rend au «jardin de granit» (59) près de Ouagadougou où les symposiums de sculpture de Sy Kiriky transforment les pierres de granit exposées au soleil du désert en oeuvres d'art. Tous les éléments évoqués, aussi nombreux soient-ils, s'inscrivent dans un ensemble plus grand, celui de l'éveil de l'Afrique: «Ici, l'inertie naturelle a été bouleversée, la beauté créée. Un destin figé, tel ces blocs de granit, est devenu un destin construit, une préfiguration de ce que pourrait être, ce que sera le continent» (62).

Du village de Mbingou, au Gabon, et l'atelier-coopérative où Pierre, enfant sculpteur «comblé d'un don secret, héritage du peuple Akellé» (64) façonne «de ses mains minuscules un fin visage de pierre à la somptueuse coiffure» (67), Jean-Louis Roy nous entraîne à Kinshasa, dans des casernes désaffectées, à l'ombre du palais du Maréchal-Président, où sont conservées par Lema Gwete les collections d'oeuvres d'art des peuples du Zaïre, «des beautés venus des siècles, des lieux intimes de la croyance et de la ferveur, des zones immatérielles où cohabitent les espérances les plus nourricières et les craintes qui leur sont inséparables» (70). Puis il rappelle l'Académie malgache d'Antananarivo, institution libre, lieu de parole où des générations d'hommes et de femmes ont «lanc[é] dans l'espace commun "des oiseaux immatériels" en espérant qu'ils fassent leur nid dans la conscience affirmée contre l'inconscience ambiante» (76). À Casablanca, il nous fait part des interrogations que pose la revue Prologues dont le but est d'«éclairer et dépasser "l'espace d'angoisse dans lequel se débat la conscience des musulmans contemporains"» (82) écartelée entre la tradition musulmane et la modernité, et il évoque l'avenir du Maroc face à la mondialisation des échanges. Du «El Hadj Taya» à Niamey, école de musique et centre de promotion de musiques africaines, où l'accueille la conservatrice Mariama Hima, il nous emmène à Dioïla, à cent cinquante kilomètres de Bamako, où l'on inaugure une bibliothèque consacrée aux ouvrages en langues 
nationales africaines.

Certes, tous ses interlocuteurs parlent français, mais comme le fait remarquer l'auteur, «les langues occidentales sont ici eaux lointaines peu susceptibles d'assouvir la soif quotidienne et d'éteindre l'incendie proche. Elles doivent consentir et profondément à une cohabitation déséquilibrée avec les langues africaines» (101). Ainsi, un pédagogue peut facilement discuter en bambara de ses recherches et de sa thèse doctorale consacrées à Montaigne (101), car l'Afrique demeure plurilingue, «capable des plus exigeantes plongées dans les langues occidentales mais vivant au quotidien l'aisance naturelle dans ses langues ancestrales» (101). Et que dire des griots qui témoignent «d'une véritable civilisation de l'oralité capable de conserver des récits souvent anciens et d'une surprenante précision» (108) dans des langues souvent non écrites? La question des langues nationales, facteur de cohésion des peuples africains, demeure entière et l'auteur rappelle en quelques pages toute l'étendue de la problématique de l'«héritage pléthorique» (133) de certains pays: «206 langues au Zaïre, 185 au Cameroun, 60 au Burkina Faso, 58 au Bénin, 12 au Mali...» (113). Dans un tel contexte, qui y a-t-il de plus normal que de parler de Montaigne à ses amis... en bambara.

Enfin, dans un ultime chapitre, l'auteur évoque la fin du «soleil de mort» (116) qui planait sur Conakry, en Guinée, la fin de la dictature de Ahmet Sékou Touré, et comment «la parole inquiète [...] incertaine apprenait à nouveau les premiers mots, se figeait à les entendre, risquait de courtes phrases» (117) dans le lent apprentissage d'une liberté nouvellement acquise. Dans tous ces récits, Jean-Louis Roy témoigne de la prise d'initiatives d'individus et de groupes, «du bonheur évident de ceux qui, dans des conditions objectivement ardues poussent loin le désir de ruiner la fatalité» (14). Il cherche à décrire une Afrique culturellement en éveil, à montrer cette faim d'interrogation, de libération et de rédemption qui l'anime, à l'encontre de cette Afrique qui a été mise à l'écart de la famille humaine, humiliée, colonisée et découpée au siècle dernier et à l'encontre de ceux pour qui l'Afrique est plongée dans une nuit implacable avec ses dictatures, sa corruption et ses massacres interethniques. Pendant trop longtemps, le Sujet occidental s'est gargarisé d'images dévalorisantes de l'Autre: elles ont alimenté le sentiment de supériorité que lui donnait son rapport à la science et à l'espace, et ont joué un rôle idéologique dans sa politique de domination. Comme le répète Jean-Louis Roy à maintes reprises, «il est difficile de parler normalement de l'Afrique» (16 et al), en grande partie à cause du «poids des vérités incrustées dans l'histoire» (26), mais l'auteur prend soin de garder les yeux grands ouverts pour tout regarder, pour tout voir, surtout pour ne rien manquer de l'effervescence qui anime ce continent unique et pluriel, fascinant dans ses moindres replis.

Ce regard sur les choses dans Lumières d'Afrique n'atteindrait qu'un pittoresque superficiel s'il n'en perçait l'enveloppe pour parvenir à une réalité plus essentielle, qu'il s'agisse des formes diverses de la vie sociale africaine ou de l'apport irremplaçable de chaque être rencontré. Jean-Louis Roy refuse le regard froid du clinicien afin de privilégier l'émotion, les

\section{LittéRéalité 84}


réflexes du coeur. Les conversations sont ferventes, l'amitié au menu, la parole abondante, l'auteur succombe, selon le mot de Rilke, «au besoin et au plaisir de confiance» (31). Il refuse de dominer les hommes qu'il rencontre, bien qu'il soit lui-même haut diplomate, et choisit de les accompagner, d'être avec eux et l'un d'eux. Il sait qu'en «chaque personne se retrouve la flamme invisible de l'autre» selon l'admirable synthèse de Nourreddine Aba (27). L'altérité n'est pas l'imposition à l'Autre d'une idéologie, mais la reconnaissance d'une interrogation commune, partageable. Ce faisant, son livre acquiert une qualité de témoignage remarquable et dévoile la diversité et la richesse des rencontres et des échanges.

Écrire le voyage, c'est transformer l'expérience en conscience, selon le mot de Malraux. Après avoir parcouru ces oeuvres de Jean-Louis Roy, le lecteur ne peut que remettre en question les lieux communs rebattus, prétentieux, que l'on porte sur cette civilisation différente qui est celle du continent africain. L'auteur évoque une réalité nouvelle, révèle une connaissance de l'intérieur, et non une construction essentiellement imaginaire, un fantasme entretenu de l'Afrique, terre inconnue, terre méconnue. De même, la progression dans l'espace géographique africain s'accompagne d'une progression dans la réalité humaine. S'inscrit à travers ces oeuvres, le vieux rêve du voyageur qui est toujours, on le sait depuis Montaigne, de mieux percevoir, en se frottant aux façons de vivre et de penser et aux usages les plus divers, le caractère relatif et arbitraire de sa propre pratique quotidienne, et par là même d'en arriver à une conception plus vaste et plus compréhensive de la réalité humaine. Ce regard sur l'Autre que porte Jean-Louis Roy est, somme toute, un regard sur soi car, comme il le résume admirablement, «qui reste insensible à cette diversité ambulante est condamné à ne rien comprendre de l'Afrique et des Africains, à ne rien comprendre de lui-même» (50).

\section{Notes}

1 Expression du Mauricien Edouard J. Maunick pour qualifier Jean-Louis Roy, «Préface», Lumières d'Afrique de Jean-Louis Roy, [Dakar], Les Éditions Feu de brousse, [1997], p.7.

2 Jean-Louis Roy, «Douceur de la terre», Terre féconde, Montréal, Leméac, 1979, p.135.

3 Jean-Louis Roy, Le Pèlerin noir, Montréal, Hurtubise HMH, 1997, 178 p.

${ }^{4}$ L'auteur a publié dix-sept livres, dont un roman: La Beauceronne, Marie à Georges à Joseph (1977), un recueil de nouvelles: Des vies et des fleuves (1995) et quatre recueils de poésie: Les Frontières défuntes (1972), Rameaux du vieil arbre (1973), L'Arche dans le regard (1975) et Terre féconde (1979).

${ }_{5}$ Jean-Louis Roy, Lumières d'Afrique, [Dakar], Les Éditions Feu de brousse, [1997], 124 p. 